\title{
Study on Method to Generate a Virtual Lane using V2V-based Clothoid Road Model
}

\author{
Hyeonggeun Mun ${ }^{1}$, Ming Lin ${ }^{1}$, Byeongwoo Kim ${ }^{2}$ \\ ${ }^{I}$ Dept. of Electrical Engineering, Ulsan University \\ 93 Daehak-ro, Mugeo-Dong, Nam-Gu, Ulsan 680-749, KOREA \\ mhg0005@gmail.com, flaaud159@gmail.com \\ ${ }^{2}$ Dept. of Electrical Engineering, Ulsan University \\ 93 Daehak-ro, Mugeo-Dong, Nam-Gu, Ulsan 680-749, KOREA \\ Corresponding Author: bywokim@ulsan.ac.kr
}

\begin{abstract}
This study proposes a way to generate a virtual lane by using a vehicle's driving trajectory during the operation of a V2V-based vehicle. The existing method of road shape detection using Lidar, Radar, and camera sensors has limitations in the range of detection with blind spots. To overcome such limitations, a method to generate virtual lanes was proposed to detect the road shape along with the driving trajectory of the leading vehicle based on V2V communications. A Clothoid road model was applied for the calculation of the driving trajectory of the leading vehicle. A driving scenario with various changes in curvature of road was also configured. Furthermore, the feasibility of the virtual lane generation method was verified by comparing the reference virtual lane and the virtual lane generated by the driving trajectory. The simulation results confirmed the usability of the virtual lane generated from Clothoid road model.
\end{abstract}

Keywords: Clothoid Road Model, Curvature, V2V (Vehicle to vehicle), Path Tracking, Virtual Lane

\section{Introduction}

Interest in studying intelligent vehicles is recently growing. In particular, the high-tech active safety system for collision prevention between vehicles is under active research. The active safety system such as LDWS (Lane departure warning system), LKAS (Lane keeping assist system), BSD (Blind spot detection), AEB (Autonomous emergency braking), FCW (Forward collision warning), or RCW (Rear-end collision warning) is mounted to commercial vehicles after considerable development. The currently existing active safety system detects the target using Lidar, Radar, or Camera sensors [1][2]. The system using sensors not only has a limited range of detection on curved roads or intersections, but also cannot detect areas of blind spots.

To overcome such restrictions, studies on active safety systems using V2V (Vehicle to vehicle) or V2I (Vehicle to infra) communication are now being carried out [3][4]. Because the vehicle information is exchanged via wireless communication between vehicles, the sensor limit for its detection range and blind spots can be resolved.

To achieve more accurate control over the high-tech active safety system using communication, it is important to understand the shape of the road on which the vehicle is being driven [5]. Existing studies have shown the method of detecting and generating lanes using lidar, radar, and camera sensors [6][7]. This study used the information of the V2V-based leading vehicle to generate its vehicle trajectory. Furthermore, virtual lanes were generated using the driving trajectory to identify the road shape, verifying situations 
with collision risk, as well as selection and control of the right high-tech active safety system.

B-spline curve and Clothoid curve are typically used for generating the curve trajectory [8][9][10].

B-spline curve method generates an accurate trajectory as it positions multiple locations spots and then creates the driving trajectory. The Clothoid curve method first creates the trajectory according to the road curvature, which is calculated by the location information and in-vehicle information.

$\mathrm{B}$-spline curve method requires the user to determine the degree of the trajectory function and the control point. A fluctuating function appears when the degree of the trajectory function increases, and the operation quantity increases if the degree of the function and the control point are set high. Thus, the degree of function, as well as the control points, restrictively affects its performance. As using equations of higher operation quantity in a real autonomous navigation vehicle is difficult, the Clothoid curve of rather simple formula and less computation was used in place of the B-spline curve.

To identify the road shape, a virtual lane was generated by the driving trajectory. The generated virtual lane was applied with left and right offsets from the road width regulations of Korea.

This paper proposes a method to generate a virtual lane using the Clothoid road model in a $\mathrm{V} 2 \mathrm{~V}$ environment.

\section{Virtual Lane Generation Method}

\subsection{System Configuration}

Figure 1 illustrates the flowchart of the virtual lane generation system. The vehicle location information $\mathrm{X}$ and $\mathrm{Y}$, and the in-vehicle data $\varphi$ (Yaw rate) and $\mathrm{v}$ (velocity), can be obtained by the in-vehicle sensor. Input for the Clothoid road model is the vehicle location and in-vehicle information. The output for the Clothoid road model is the vehicle's driving trajectory. Therefore, the vehicle's driving trajectory can be found by the Clothoid road model. To generate the virtual lane, the Korean road design standard ( $3.5 \mathrm{~m}$ wide) was considered for the left and right offsets centered at the driving trajectory. The virtual lane can be created by the vehicle's heading angle and its driving trajectory.

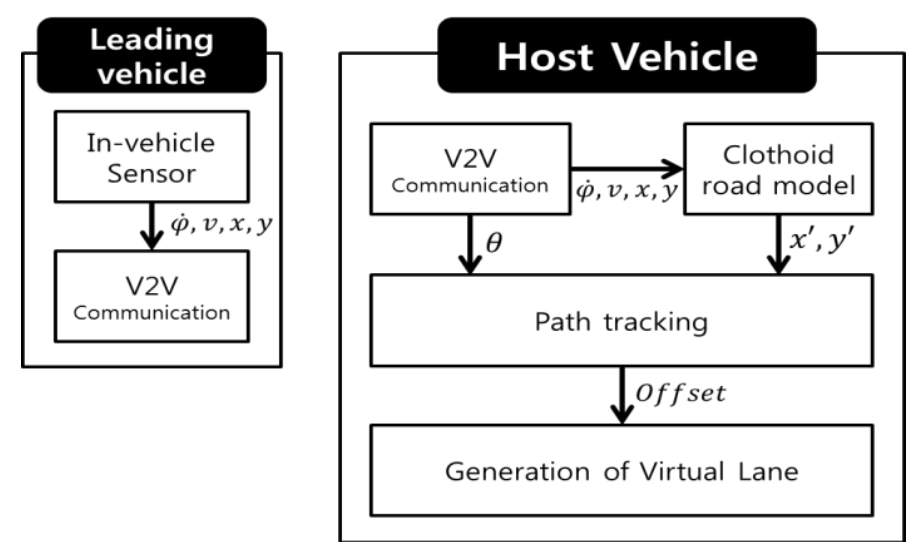

Figure 1. Flow Chart of the Virtual Lane Generation System

\subsection{Clothoid Road Model}

A vehicle's driving trajectory is needed to generate a virtual lane. The driving trajectory was created from the equations from Clothoid road model, which uses the road curva- 
ture for its calculation. Equation (1) shows the formula to obtain the curvature. Here, $\mathrm{K}$ represents the curvature and $\mathrm{R}$ for the radius of curvature. Equation (2) shows the formula to obtain the radius of curvature, which uses the velocity of the leading vehicle and its Yaw rate for the calculation. The reciprocal of the obtained radius of curvature leads to the curvature of the driving trajectory.

Equation (3) means the change in tangent angle between two coordinates. The change in tangent angle can be calculated by integrating the curvature and distance between two coordinates. It can be represented by the difference of the tangent angle at the point to that at the other point, $\theta(\mathrm{l})$ and $\theta_{0}$. In addition, the difference of the two tangent angles can be expressed as the integration equation by the distance between the two points. The right side of Equation (1) describes curvature. It can be represented by adding the curvature rate to the current value. Here, $\mathrm{C}_{0}$ is curvature, $\mathrm{C}_{0}$ is the curvature rate, and ${ }_{-} \mathrm{l}_{-}$the distance between two points. Equation (4) transforms the tangent angle in the polar coordinate system to that in the Cartesian coordinate system. The $\mathrm{x}$-axis and the-y-axis were set according to the Earth's absolute coordinate system. The x-coordinate and y-coordinate can be obtained by adding the change in the distance between the two points to the current coordinate $(\mathrm{x} 0, \mathrm{y} 0)$.

Equation (5) is the final Clothoid road model equation represented on the Cartesian coordinate system. The $\mathrm{x}$-coordinate as well as the y-coordinate is calculated using curvature, change in curvature, and distance between the two points. It was obtained by the Taylor expansions of $\sin \theta$ and $\cos \theta$ in Equation (4). The vehicle drives along the trajectory and calculates the $\mathrm{x}$ - and $\mathrm{y}$-coordinate for the two absolute coordinates, drawing the driving trajectory.

$$
\begin{aligned}
& K=\frac{1}{R}=C_{0}+C_{1} l \\
& R=\frac{v}{\dot{\varphi}} \\
& \Delta \theta(l)=\theta(l)-\theta_{0}=\int_{0}^{l} K(t) d t=C_{0} l+\frac{1}{2} C_{1} l^{2} \\
& \left\{\begin{array}{l}
x(l)=x_{0}+\int_{0}^{l} \cos \theta(t) d t \\
y(l)=y_{0}+\int_{0}^{l} \sin \theta(t) d t \\
x(l)=l \\
y(l)=\frac{1}{2} C_{0} l^{2}+\frac{1}{6} C_{1} l^{3}
\end{array}\right.
\end{aligned}
$$

\section{Simulation and Results}

Figure 2 illustrates the simulation system configuration environment. The PreScan tool was used to configure the $\mathrm{V} 2 \mathrm{~V}$ communication environment and to model the road shape 
and driving conditions. MATLAB/Simulink tool was used for running the Clothoid road model and virtual lane generation algorithm. The entire system was configured by interconnecting PreScan and MATLAB/Simulink.

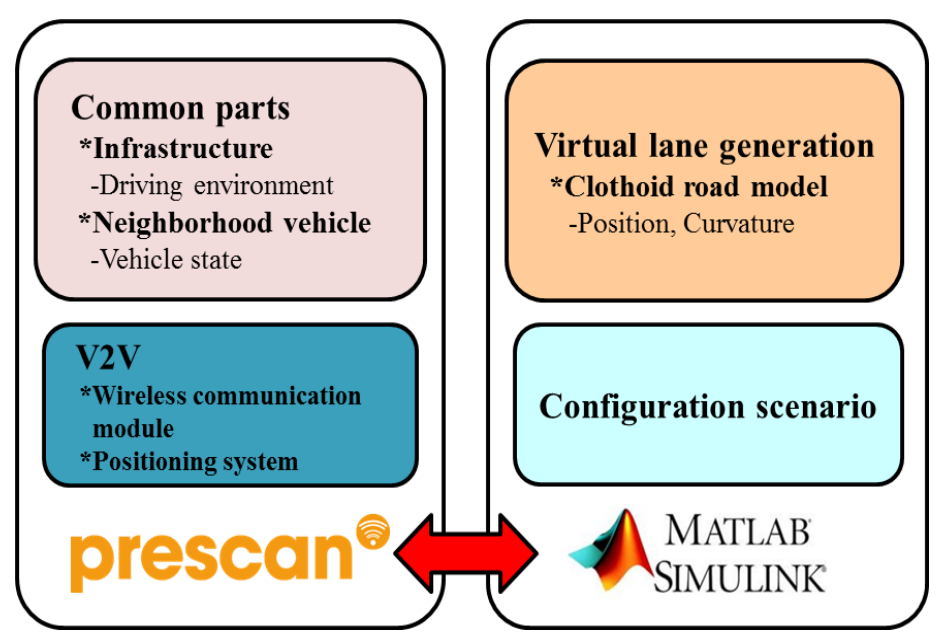

Figure 2. Simulation System Configuration

\subsection{Simulation Scenario}

The driving scenario was created as in Figure 3 to generate a virtual lane from the driving trajectory on a road. The leading vehicle operates at a constant speed of $60[\mathrm{~km} / \mathrm{h}]$. The host vehicle receives location and velocity of the leading vehicle as well as its Yaw rate information through $\mathrm{V} 2 \mathrm{~V}$ communication. The leading vehicle and the host vehicle communicate with $\mathrm{V} 2 \mathrm{~V}$ at an interval of $0.01[\mathrm{~s}]$. The location information of the vehicle is received by the target vehicle as absolute information. The driving trajectory is generated by the information from the leading vehicle, and therefore, a virtual lane is generated to detect the road shape.

The curvature of the road model was divided into three cases: no change in curvature, constant change in curvature, and non-constant changes in curvature. In addition, the width of the virtual lane was set as $3.5[\mathrm{~m}]$ to meet the current Korean road design regulations.

The driving simulation was carried out depending on the pre-set velocity conditions of the leading vehicle. Thus, the virtual lane was generated from the driving trajectory of the V2V-based leading vehicle. 


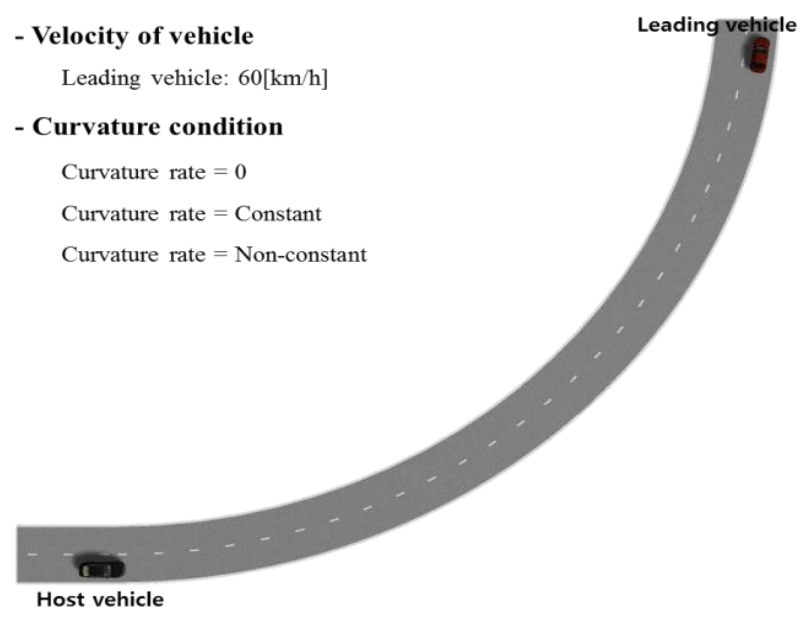

Figure 3. Driving Scenario for Simulation

\subsection{Simulation Results}

Scenarios defined in 3.1 confirm the results of the virtual lane generation simulation according to its driving trajectory.

Figure 4 represents the curvature rate of the simulation road model. The curvature rate rapidly changes at about $0.3[\mathrm{~s}]$ in Figure 4 . This is because the vehicle velocity and Yaw rate sharply shift at the beginning of the simulation. Bend curve road is the road model with constant curvature. As the velocity and the Yaw rate change at a constant rate on the road with constant curvature, the curvature rate turns out to be 0 . Therefore, the curvature rate appears as 0 in Figure 4, Curvature rate $=0$ road model. The Clothoid curve road is the road model with a constantly changing curvature. As the operating velocity of the vehicle is constant with large Yaw rate change, the curvature increases. With constantly increasing curvature, the curvature rate becomes constant. Therefore, Figure 4 shows the constant curvature rate with the value of $0.01[\mathrm{~m}-1 / \mathrm{s}]$. A Beizer curve road is the road model of non-constant curvature. The change in Yaw rate increases inconsistently on curved roads. As the Yaw rate does not increase at a constant rate, the curvature value is also not consistent. As the curvature is not consistent, the curvature rate increases over time. Therefore, the rising curvature rate is shown in Figure 4. 


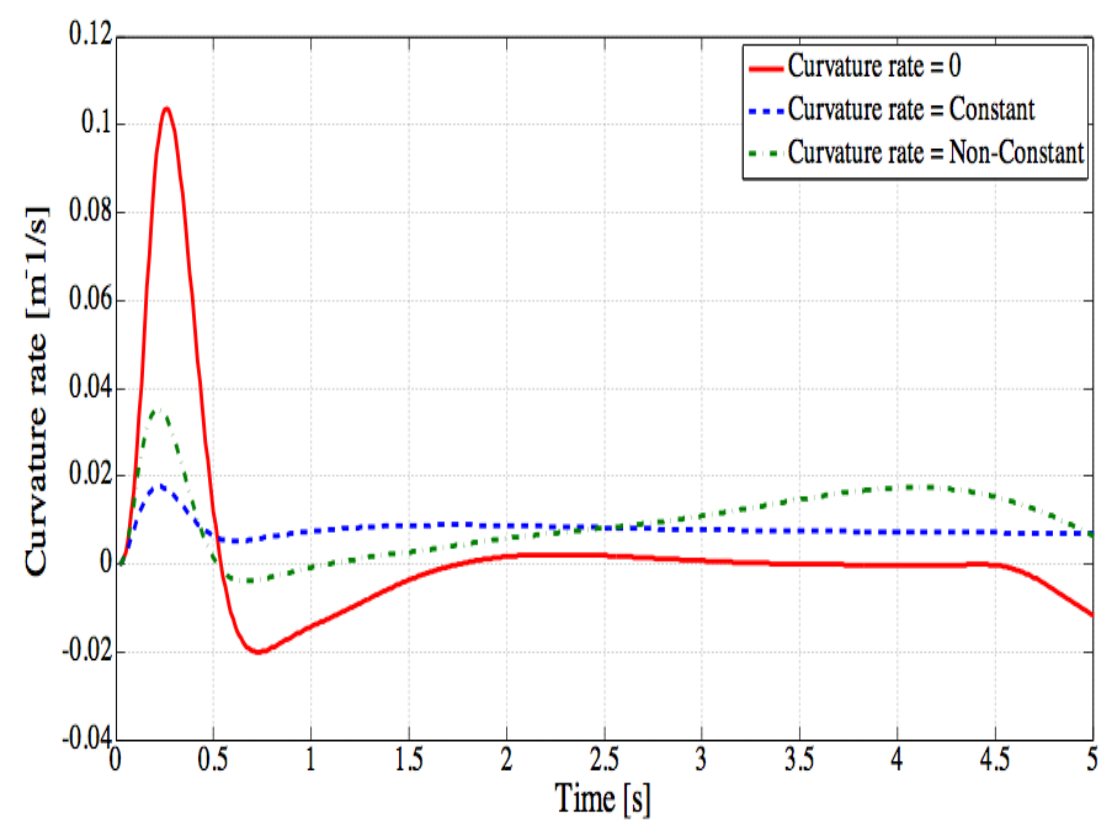

Figure 4. Curvature Rate of the Simulation Road Model

Figure 5 illustrated the virtual lane generated according to the driving trajectory calculated from the leading vehicle information. Figure 5-(a) shows the road model with 0 curvature rate. At the start point, the reference trajectory is confirmed to closely match the reference and simulation trajectory. This is because there is no yaw rate change on a straight road. Moreover, when the operating vehicle enters the curved area, the yaw rate sharply changes, creating error to the reference trajectory. Because the road model in Figure 5-(a) has a constant rate of yaw rate change, its error is smaller than that in Figures 5(b) and 5-(c). Figure 5-(a) helps to verify that the error to the reference trajectory occurs in the curved area of the road. The road model in 5-(b) has a constant curvature rate. Its error to the reference trajectory appears because of the constant velocity and the increasing rate of the yaw rate change in the curved area. The error size is bigger as the yaw rate change is larger than that of the Figure 5-(a) road model. The road model of Figure 5-(c) has an inconsistent curvature rate. With its largest curvature rate, the rate of yaw rate change is also the largest, which produces the largest curvature rate from Equation (1). Therefore, it creates the largest error to the reference trajectory among the three road models.

The similarity between the reference driving trajectory and the trajectory calculated from the Clothoid road model can be confirmed in the three scenarios. In addition, a virtual lane was generated by adding a $1.75[\mathrm{~m}]$ offset on the left and right to the calculated road trajectory at the center. The generated virtual lane closely overlapped the reference lane. Therefore, we confirm that the generated virtual lane has similar shape to that of the reference lane. 


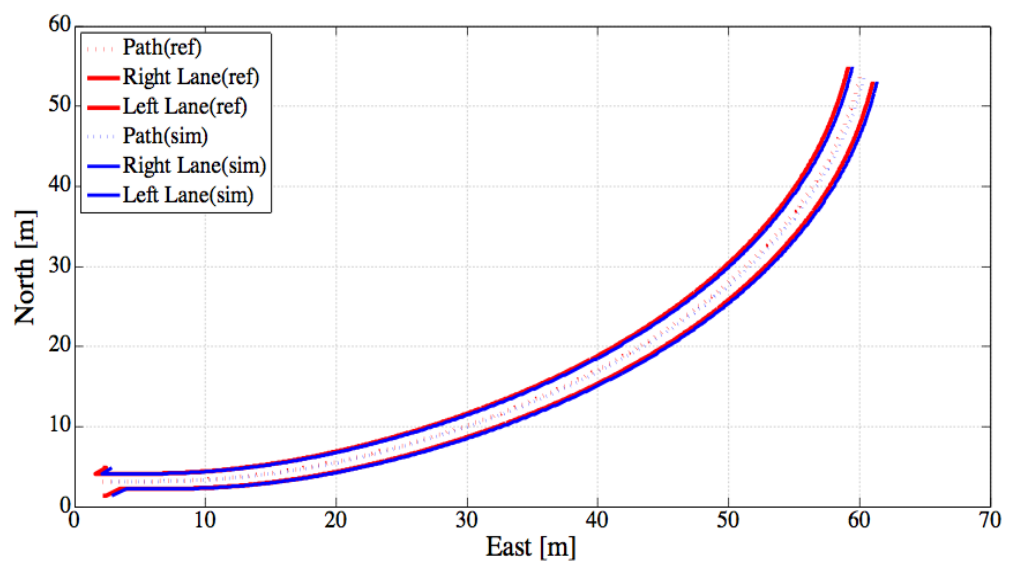

(a)

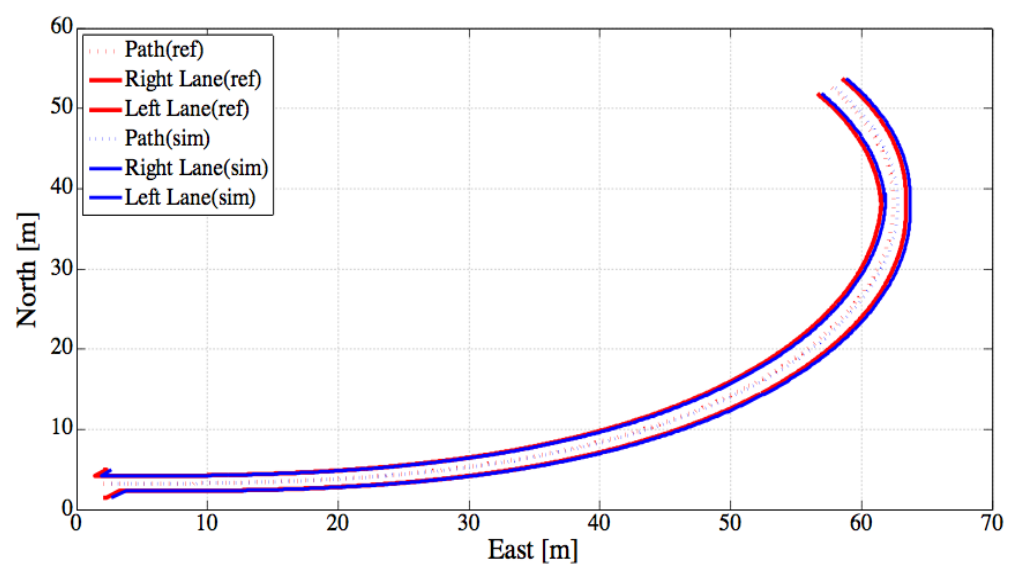

(b)

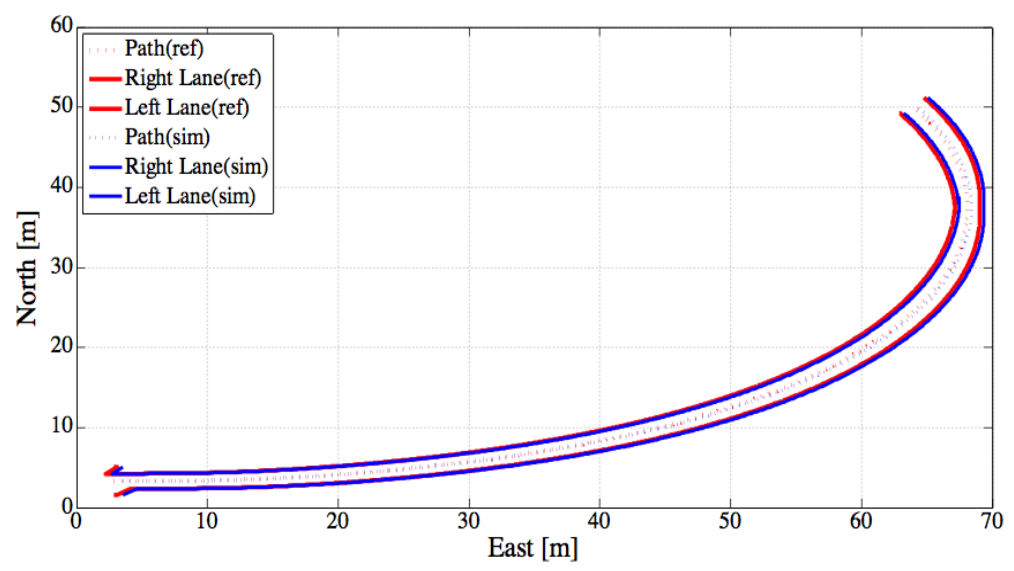

(c)

\section{Figure 5. Virtual Lane Generation to the Driving Trajectory on Road}

Figure 6 shows the error of the driving trajectory calculated from the Clothoid road model to the reference model. The error size was represented as the difference in distance between the reference driving trajectory coordinates and calculated driving trajectory coordinates. With a constant velocity of the leading vehicle and the changing yaw rate for each driving road model, the largest error size appears in the road model with the largest curvature rate. The road with 0 curvature rate has a uniform yaw rate and a constant driving velocity, which produces the smallest error size. 
Table 1 displays the average error size of road models with different curvature rate. The average error size of the road model with 0 curvature rate is calculated as $0.32[\mathrm{~m}]$, that of the road model with constant curvature rate is $0.34[\mathrm{~m}]$, and lastly, the average error size of the road model with non-constant curvature rate is $0.35[\mathrm{~m}]$. Because the driving velocity of the leading vehicle is consistent in all three road models, the parameter that affects the curvature is the yaw rate. The largest average error size appears in the road with the biggest curvature rate, as the yaw rate also largely changes.

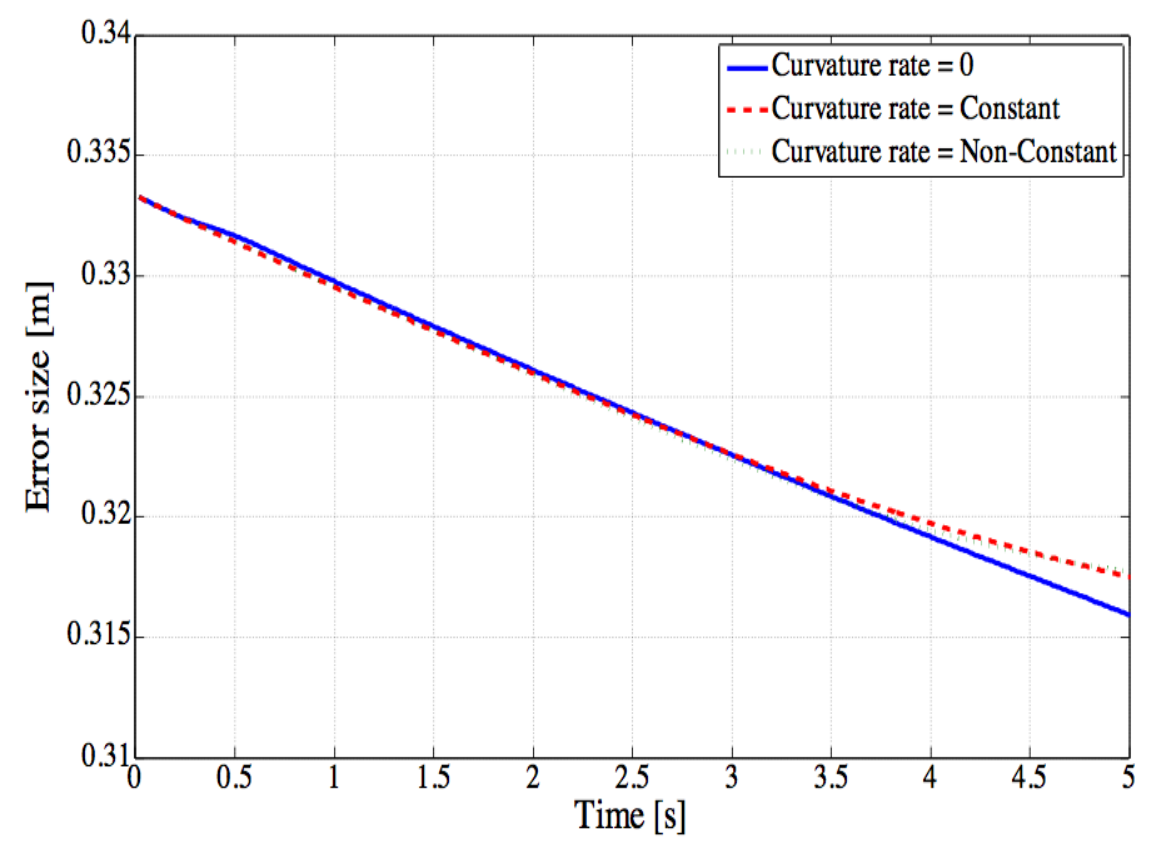

Figure 6. Difference in Trajectory Error Size of the Clothoid Road Model and the Reference

Table 1. Average Error size of the Driving Trajectory

\begin{tabular}{|c|c|c|c|}
\hline Curvature rate $\left[\mathrm{m}^{-1} / \mathrm{s}\right]$ & Zero & Constant & Non-constant \\
\hline Average error size $[\mathrm{m}]$ & 0.32 & 0.34 & 0.35 \\
\hline
\end{tabular}

\section{Conclusion}

In this paper, we studied a method to generate virtual lanes, carried out by using the V2V-based Clothoid road model in order to identify the road shape. Based on the V2V communication, the proposed virtual lane generation system generated virtual lanes for the driving trajectory on several road models using location and velocity of the leading vehicle and the yaw rate. The Clothoid road model was utilized to generate the driving trajectory on-road. The curvature parameter needed for the Clothoid road model was calculated from the leading vehicle's velocity and yaw rate. The virtual lane was created by setting the left/right offset to the generated driving trajectory.

To verify the feasibility of the proposed virtual lane generation, several simulations were performed on various road models. Three scenarios were applied to the road model: no curvature rate, and constant and non-constant curvature rate. The simulation results 
confirm that the driving trajectory as well as the virtual lane was generated similarly to the road shape model. The average error size of the simulation driving trajectory to the reference was confirmed as $0.32[\mathrm{~m}]$ on the road model with no curvature rate, $0.34[\mathrm{~m}]$ with constant curvature rate, and $0.35[\mathrm{~m}]$ with non-constant curvature rate.

This paper proposes a method to generate virtual lanes using a V2V-communicationbased Clothoid road model and its usability was verified by the simulation on several road models. Future study will be concerned with the method of generating a driving trajectory on the road, depending on the vehicle's location noise.

\section{Acknowledgments}

This work was supported by the Ministry of Trade, Industry and Energy(MOTIE) Institutional Pro-gram(Project No. 10043816, "The Development of the High-Definition 3DHDR Camera and the Integrated Image Processing Module for Parking/Lane Changing Driver Assistance Systems.").

This work was supported by the IT Research and development program of The MOTIE/KEIT(Ministry of Trade, Industry and Energy/Korea Evaluation Institute of Industrial Tech-nology) [N0000889, The infrastructure build of wireless and application service evaluation - certification for cooperative vehicular safety systems.]

\section{References}

[1] T. D. Gamage, J. G. Samarawickrama and A. A. Pasqual, "GPU based non-overlapping multi-camera vehicle tracking", IEEE 7th International Conference on Information and Automation for Sustainability, (2014)

[2] C. Wei, Y. Zeng and T. Wu, "Obstacle Detection Based on a 2D Large Range Sonar Model", IEEE 6th International Congress on Image and Signal Processing (CISP 2013), (2013), pp.1127-1131.

[3] N. Kim, J. Lee, M. Soh, J. Kwon, T. Hong and K. Park, "Improvement of Longitudinal Safety System's Performance on Near Cut-In Situation by Using the V2V", Korean Society of Automotive Engineers, (2013), pp.747-755

[4] B. Xie, K. Li, X. Qin, H. Yang and J. Wan, "Approaching Index Based Collision Avoidance for V2V Cooperative Systems", IEEE 17th International Conference on Intelligent Transportation Systems, (2014), pp. 127-132.

[5] J. Park, C. Kim, S. Bai, J. Lee, T. Chung and K. Huh, "Development of The Lane Detection System based on V2V Communication", The Korean Society Of Automotive Engineers, (2014), pp.595-597.

[6] Y. Chen and M. He, "Sharp Curve Lane Boundaries Projective Model and Detection", IEEE 10th International Conference on Industrial Informatics, (2012), pp.1188-1193.

[7] J. Yang, Z. Li and L. Li, "Lane Detection Based on Classification of Lane Geometrical Model", IEEE 11th International Conference on Signal Processing (ICSP), (2012), pp.842-846.

[8] K. Jo and M. Sunwoo, "Generation of a Precise Roadway Map for Autonomous Cars", IEEE Transactions on Intelligent Transportation System, (2014), pp. 925-937.

[9] L. B. Cremean and R. M. Murray, "Model-Based Estimation of Off-Highway Road Geometry using Single-Axis LADAR and Inertial Sensing", IEEE International Conference on Robotics and Automation, (2006), pp.1661-1666.

[10] H. Mun, M. Lin and B. Kim, “A Virtual Lane Generation Method in a V2V Environment”, Asia-pacific Proceedings of Applied Science and Engineering for Better Human Life, (2016), pp.45-49.

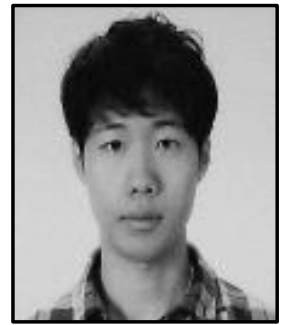

Hyeonggeun Mun, he received his B.E. degree in University of Ulsan in 2015, Ulsan, South Korea. Now he is pursuing his M.E. degree in electrical engineering in University of Ulsan, Ulsan, South Korea. His current research interests include autonomous emergency braking (AEB) system, vehicle to vehicle (V2V) Communication and advanced driving assistance system (ADAS). 

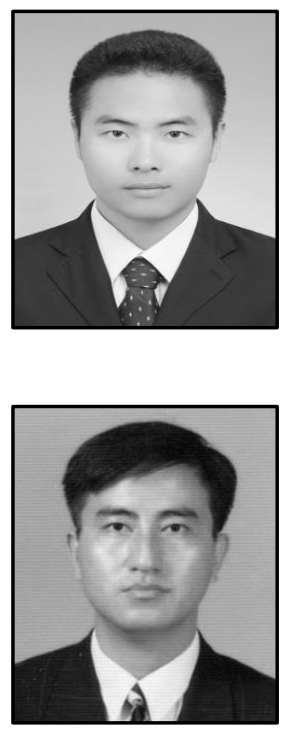

Ming Lin, he received the bachelor degree in Shanghai University of Engineering Science, Shang Hai, China. He is pursuing his master degree in electrical engineering in University of Ulsan, Ulsan, South Korea. His research interests include advanced driving assistance system (ADAS), and autonomous emergency braking (AEB) system.

Byeongwoo Kim, he received the B.E, M.E. and Ph.D degree in Precision Mechanical Engineering from Hanyang University. He worked at KOSAKA Research Center in 1989. He worked at KATECH electrical technology Research Center from 1994 to 2006. Now he is a professor in the School of electrical engineering in University if Ulsan, Ulsan, South Korea from 2006. His current research interests include advanced driving assistance system (ADAS), and autonomous emergency braking (AEB) system. 